\title{
The expression and clinicopathological significance of CD56, CDX2, and LRP16 in intrahepatic cholangiocarcinoma
}

\author{
Zhanbo Wang ${ }^{1 \#}$, Haowen Tang ${ }^{2,3 \#}$, Jing Yuan $^{1}$, Xin Song $^{1}$, Zhigang Song $^{1}$, Po Zhao ${ }^{1}$ \\ ${ }^{1}$ Department of Pathology, ${ }^{2}$ Department of Hepatobiliary Surgery, Chinese PLA General Hospital, Beijing 100853, China; ${ }^{3}$ Graduate School of \\ Medicine, The University of Tokyo, Tokyo, Japan \\ Contributions: (I) Conception and design: H Tang, Z Wang, P Zhao; (II) Administrative support: J Yuan; (III) Provision of study materials or patients: \\ X Song; (IV) Collection and assembly of data: H Tang, Z Song; (V) Data analysis and interpretation: Z Wang, H Tang, P Zhao; (VI) Manuscript \\ writing: All authors; (VII) Final approval of manuscript: All authors. \\ \#These authors contributed equally to this work. \\ Correspondence to: Po Zhao. Department of Pathology, Chinese PLA General Hospital, Beijing 100853, China. Email: zhaopo301@yeah.net.
}

\begin{abstract}
Background: To explore the expression and clinicopathological significance of CD56, CDX2, and LRP16 in intrahepatic cholangiocarcinoma (ICC).

Methods: Immunohistochemistry for CD56, CDX2, and LRP16 was performed in 127 cases of ICC. Medical records were reviewed and clinicopathological analysis was performed.

Results: CD56 expression was detected in 27 cases (21.3\%); CDX2 staining was observed in 33 cases (26.0\%); and LRP16 expression was noted in 119 ICC specimens (93.7\%). Meanwhile, LRP16 expression was significantly in negative correlation with both CD56 and CDX2 expression. Furthermore, positive expression of CD56 or CDX2 were significantly linked with longer overall survival (OS) for ICC patients; LRP16 positive expression patients had a median survival of 20.2 months, whereas those with negative expression featured a median survival of 74.5 months $(\mathrm{P}<0.001)$.
\end{abstract}

Conclusions: CD56 positive expression, CDX2 positive expression or LRP16 negative expression implied significant survival benefits for ICC patients.

Keywords: CD56; CDX2; LRP16; immunohistochemistry; survival; clinicopathological characteristics

Submitted Mar 02, 2018. Accepted for publication Jul 17, 2018.

doi: $10.21037 /$ tcr.2018.07.18

View this article at: http://dx.doi.org/10.21037/tcr.2018.07.18

\section{Introduction}

Intrahepatic cholangiocarcinoma (ICC) arises from the epithelial lining of intrahepatic bile duct beyond the second-order of the biliary system, and constitutes approximately $10 \%$ to $20 \%$ of primary liver cancer $(1,2)$. According to anatomical location, ICC belongs to one of the three categories of cholangiocarcinoma (intrahepatic, hilar and extrahepatic). As a poorly understood variant of cholangiocarcinoma, ICC was histologically considered to be the least common category of the above three (3). The last three decades have witnessed a global increase in incidence and mortality of ICC, while the incidence of all other forms of cholangiocarcinoma has been stable or declined (4-8). The rarity of ICC poses a great obstacle not only to the understanding of disease pathogenesis but also to the development of effective treatment approaches. Surgery has been established as the mainstay of therapy and offered the hope of prolonged survival for patients with ICC. However, postoperative long-term outcome stays unsatisfactory, with 5-year survival rate of around $30 \%$ to $35 \%$ (9). Similarly, no significant survival advantages were demonstrated by locoregional neoadjuvant or palliative therapies (10-12).

With great improvement in tumor biology, bio-molecular markers from biopsy, serum sample, or postoperative specimen may be able to further predict tumor behavior. Therefore, identification of a novel prognostic predictor 
for early diagnosis, preventive measures and therapeutic regimens of ICC is of clinical utility and urgent need.

Leukemia related protein 16 (LRP16), identified and recognized as an important estrogen-responsive gene, was first isolated from human serum lymphocytes in the year 1999 (13); this novel gene was found to be on chromosome 11q12.1 and encodes nuclear factor (13-16). Increasing evidence has implied that LRP16 is closely involved in estrogen signaling pathway and can help activate estrogen receptor $\alpha(\mathrm{ER} \alpha)(17)$. Hence, LRP16 might play functional roles in tumor progression and metastasis. To date, aberrant expression of LRP16 has been found in certain primary tumors and some cell lines of breast, stomach, colon and rectum, pancreas, kidney, ovary, endometrium and prostate. Specifically, LRP16 expressions were shown to be of prognostic values in gastric carcinoma, breast cancer, colorectal carcinoma and neuroendocrine lung tumors (18-21). However, expression of LRP16 and its prognostic significance in ICC stays unexplored. Moreover, with reference to CD56 and CDX2, little data is available on the correlation of their expression with ICC outcome (association between their expression and survival outcome were not widely published, either). Given this, by using a tissue microarray approach, we evaluated immunohistochemically the expression of CD56, CDX2 and LRP16, and their prognostic values in ICC. Additionally, the associations between LRP16 expression and clinicopathological features of the included Chinese patients with ICC were also investigated.

\section{Methods}

\section{Patients and tumor samples}

The study was reviewed and approved by the Ethics Committees of the PLA General Hospital (Beijing, China). From 2005 to 2010, a total of 127 tissue samples (paraffin embedded sections) were collected from surgically resected specimens of 127 corresponding patients with pathologically confirmed ICC at the Department of Pathology, Chinese PLA General Hospital. For all the patients enrolled in the study, demographic data and preoperative presentations were collected and analyzed by reviewing the medical computerized database; the perioperative records and pathology reports were thoroughly reviewed; supplementary follow-up was carried out by telephone interviews with the patients. Patients with combined hepatocellular carcinoma and cholangiocarcinoma were excluded from this analysis.

\section{Construction of tissue microarray}

All archival formalin-fixed, paraffin-embedded sections were carefully reviewed and selected from corresponding haematoxylin and eosin stained sections to identify and mark representative tumorous areas, as previously documented $(22,23)$. Single $0.6-\mathrm{mm}$ cores of tissue taken from the abovementioned representative tumorous area were used to assemble the arrays. The assembled array blocks were sliced into 4-mmthick sections and mounted on glass slides. Totally, 127 tumor samples were evaluated for staining on the array.

\section{Immunobistochemistry}

Immunohistochemical staining of the tissues was performed using the Envision two-step method (24). Briefly, formalinfixed, paraffin wax-embedded, and 4 - $\mu$ m-thick sections were prepared as previously described (24). The slides were incubated serially (40 min each, at room temperature) with the following primary antibodies: CD56 (dilution 1:100; Gene Tech, Shanghai), CDX2 (dilution 1:150; Dako, Denmark), and LRP16 (dilution 1:150; recognized and isolated in 1999 by Department of Molecular Biology of Chinese PLA General Hospital). Each incubation with primary antibody was followed by 40 -min incubation at room temperature with horseradish peroxidase-labeled polymer. The staining was developed by incubation with diaminobenzidine substrate-chromogen for 5-10 min, and a hematoxylin counterstain was applied. Both negative and positive controls for each antibody were performed.

\section{Evaluation of score}

Immunohistochemical expression was assessed by 2 investigators using light microscopy. Both were blinded to study design. Staining was graded for intensity of staining ( 0 , negative; 1 , weak; 2 , moderate; 3 , strong) and percentage of cells stained $(0, \leq 5 \% ; 1,6-25 \% ; 2,26-50 \% ; 3,>50 \%)$. The final score was determined by the combined staining score (extent + intensity). For all the 3 antibodies, score greater than or equal to 2 was defined as positive expression (18).

\section{Definition}

In the present study, all 127 patients had received curative resection (R0 resection). Lymphatic invasion mostly referred to lymph node involvement at the site of the hepatoduodenal ligament [regional lymph nodes, N1 
Table 1 Clinicopathological characteristics and corresponding survival

\begin{tabular}{|c|c|c|c|}
\hline Variable & Cases & $\begin{array}{l}\text { Survival } \\
\text { (months) }\end{array}$ & $P$ value \\
\hline Sex & & & 0.19 \\
\hline Male & 81 & 27.0 & \\
\hline Female & 46 & 18.7 & \\
\hline Age, year & & & 0.55 \\
\hline$\leq 40$ & 15 & 18.1 & \\
\hline $41-60$ & 74 & 22.0 & \\
\hline$\geq 61$ & 38 & 29.1 & \\
\hline HBs Ag & & & 0.78 \\
\hline No & 90 & 25.2 & \\
\hline Yes & 37 & 18.8 & \\
\hline Bile duct stone & & & 0.20 \\
\hline No & 100 & 22.3 & \\
\hline Yes & 27 & 27.2 & \\
\hline Diabetes & & & 0.57 \\
\hline No & 117 & 23.9 & \\
\hline Yes & 10 & 24.3 & \\
\hline Tumor nodules & & & 0.013 \\
\hline Multiple & 33 & 14.4 & \\
\hline Single & 94 & 27.5 & \\
\hline Gross subtype & & & $<0.001$ \\
\hline Mass-forming & 75 & 24.1 & \\
\hline Peri-ductal infiltrating & 18 & 11.0 & \\
\hline Intraductal papillary & 19 & 39.3 & \\
\hline Mixed-type & 15 & 12.93 & \\
\hline Tumor necrosis & & & 0.001 \\
\hline No & 79 & 29.1 & \\
\hline Yes & 48 & 16.1 & \\
\hline Histological differentiation & & & $<0.001$ \\
\hline Well & 17 & 48.5 & \\
\hline Moderate & 28 & 28.1 & \\
\hline Poor & 82 & 15.9 & \\
\hline TNM staging & & & 0.012 \\
\hline 1 & 67 & 14.5 & \\
\hline 2 & 37 & 12.0 & \\
\hline 3 & 23 & 8.0 & \\
\hline Histological subtype & & & 0.018 \\
\hline Adenocarcinoma & 111 & 25.3 & \\
\hline Mucinous adenocarcinoma & 10 & 20.3 & \\
\hline Signet-ring cell carcinoma & 1 & 12.0 & \\
\hline Squamous cell carcinoma & 5 & 5.0 & \\
\hline
\end{tabular}

$\mathrm{P}<0.05$ was considered statistically significant. disease based on the $8^{\text {th }}$ edition of the American Joint Committee on Cancer (AJCC) staging system]. According to the macroscopic appearances proposed by the WHO Classification of Tumors of the Digestive System [2010], ICC includes four morphological growth subtypes: the mass-forming (MF) subtype, the periductal infiltrating (PI) subtype, and the intraductal growth (IG) subtype, and the mixed subtype. Morphological growth subtypes were defined based on preoperative imaging and the macroscopic description in the pathological report. Tumor histological subtypes were pathologically identified according to the predominant features.

\section{Statistical analysis}

Statistical analyses were carried out using SPSS v14.0 (IBM, Armonk, NY, USA). Categorical variables were compared by the $\chi^{2}$ test or Fisher's exact test, as appropriate. Longterm survival was estimated using the Kaplan-Meier method and compared by the log rank test. Data are the mean \pm standard deviation or median (range). $\mathrm{P}<0.05$ was considered statistically significant.

\section{Results}

\section{Demographic data and clinicopathological characteristics of the patients}

Demographic data and clinicopathological characteristics of the patients were presented in Table 1. In our survey of 127 cases of ICC, there were 81 males and 46 females; the median age was 54.85 years (range, $31-77$ years). The tumor size ranged from 1 to $24 \mathrm{~cm}$ (mean $6.47 \mathrm{~cm}$ ). As with TNM staging, there were 67 of T1 tumors, 37 of T2, and 23 of T3. Regarding histological subtypes, 122 were adenocarcinoma, and 5 were squamous cell carcinoma. As regards to histological differentiation, 17 were well differentiated, 28 moderately differentiated and 82 poorly differentiated. The follow-up length of the patients ranged from 12 to 92 months with a median of 24 months. And 16 patients lost to follow-up.

\section{Expression of CD56, CDX2, and LRP16}

Both CD56 and CDX2 staining were assessed by immunohistochemistry in all 127 ICC specimens. As illustrated in Figure $1 A$ and $1 B$, CD56 was principally concentrated in the membrane of tumor cells (Figure 1A), whereas CDX2 protein was mainly concentrated in the nucleus of tumor cells (Figure 1B). CD56 expression was 


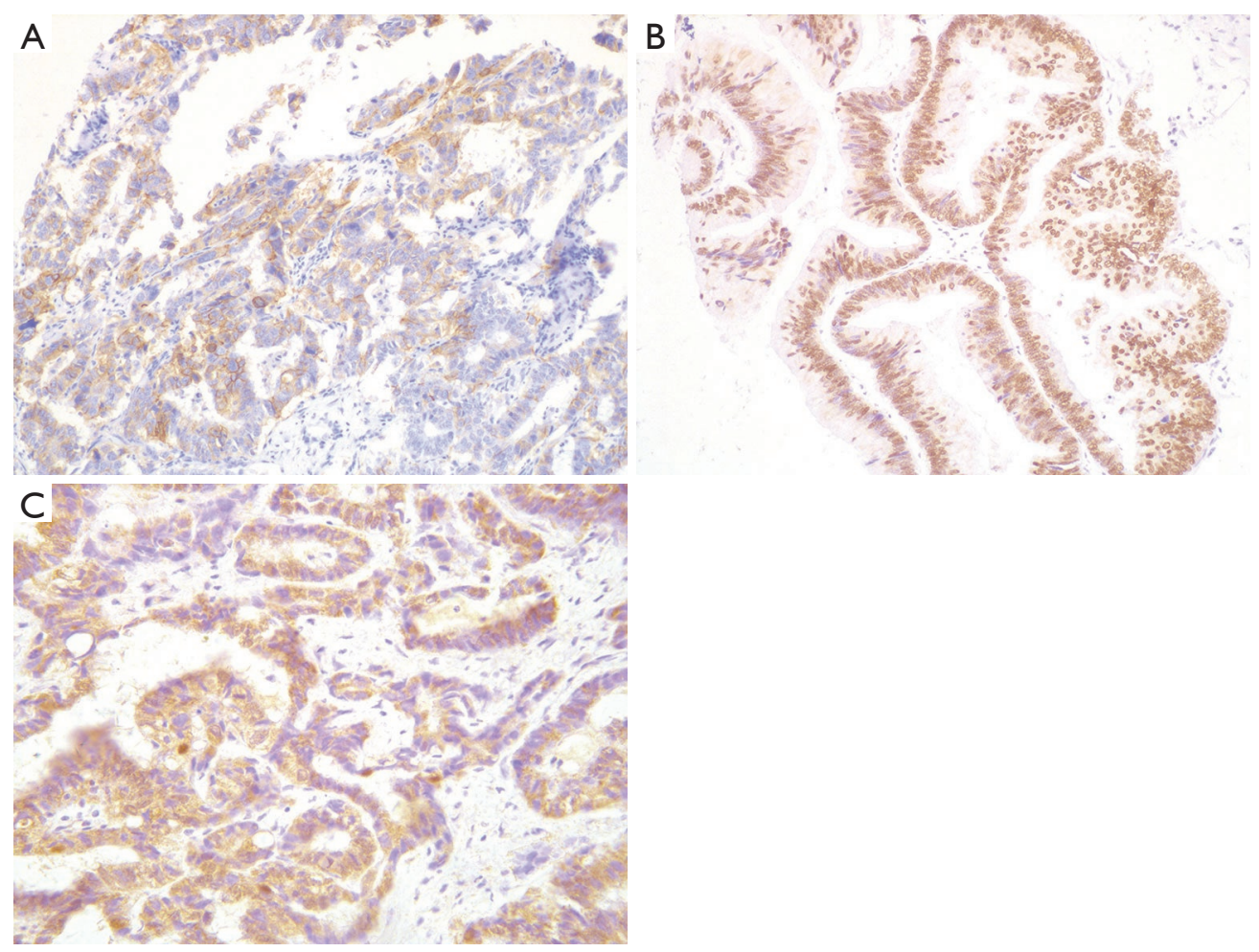

Figure 1 Immunohistochemical stainings of CD56, CDX2, and LRP16 in ICC. (A) CD56 expression in ICC. A strongly immunostained membrane of tumor cell in yellow or brown color ( $\times 200)$; (B) CDX2 expression in ICC. A strongly immunostained nucleus of tumor cell in yellow or brown color $(\times 200)$; (C) LRP16 expression in ICC. A strongly immunostained cytoplasm of tumor cell in yellow or brown color $(\times 200)$. ICC, intrahepatic cholangiocarcinoma.

detected in 27 cases (21.3\%); CDX2 staining was observed in 33 cases $(26.0 \%)$. Similarly, immunohistochemical staining of LRP16 was performed in all cases. LRP16 expression was localized mainly in the cytoplasm of tumor cells (Figure 1C) and was noted in 119 ICC specimens (93.7\%).

Further analyses revealed that LRP16 expression was negatively correlated with both CD56 (coefficient of correlation: $-0.208, \mathrm{P}=0.018$ ) and $\mathrm{CDX} 2$ expression (coefficient of correlation: $-0.218, \mathrm{P}=0.013$ ).

\section{Survival analysis}

For survival analysis, the median overall survival (OS) of the 127 patients was 12.2 month (range, 0.5-92.0 months, Figure 2A), and survival at 1,3 , and 5 years was $32 \%, 16 \%$, and $12 \%$, respectively. Patients were categorized into positive or negative groups according to the expression of the markers of interest. Patients with positive expression of CD56 had a longer median survival (39.3 months) than those with no expression (21.8 months), and the difference was statistically significant
$(\mathrm{P}=0.026$, Figure $2 B)$. Cases with positive CDX2 expression had a median survival of 41.4 months, whereas those in the negative group showed a median survival of 17.6 months $(\mathrm{P}<0.001$, Figure 2C). Of note, unlike the expression of CD56 and CDX2, the positive expression of LRP16 was associated with inferior survival when compared to negative expression (median survival: 20.2 months in positive expression group and 74.5 months in negative expression group; $\mathrm{P}<0.001$ ). Differences in 1,3 , and 5 years OS rates between positive LRP16 expression group and the negative group were also statistically significant $(28 \%$ versus $100 \%, 12 \%$ versus $88 \%$, and $9 \%$ versus $61 \%$, respectively; $\mathrm{P}<0.0001)$. Kaplan-Meier survival curves suggested that patients with negative LRP16 expression had inferior survival when compared to those with positive LRP16 expression $\left(\chi^{2}=13.2, \mathrm{P}<0.001\right.$; Figure $\left.2 D\right)$.

\section{Association between survival and other clinicopathological features}

Of the other clinicopathological features, tumor nodules 

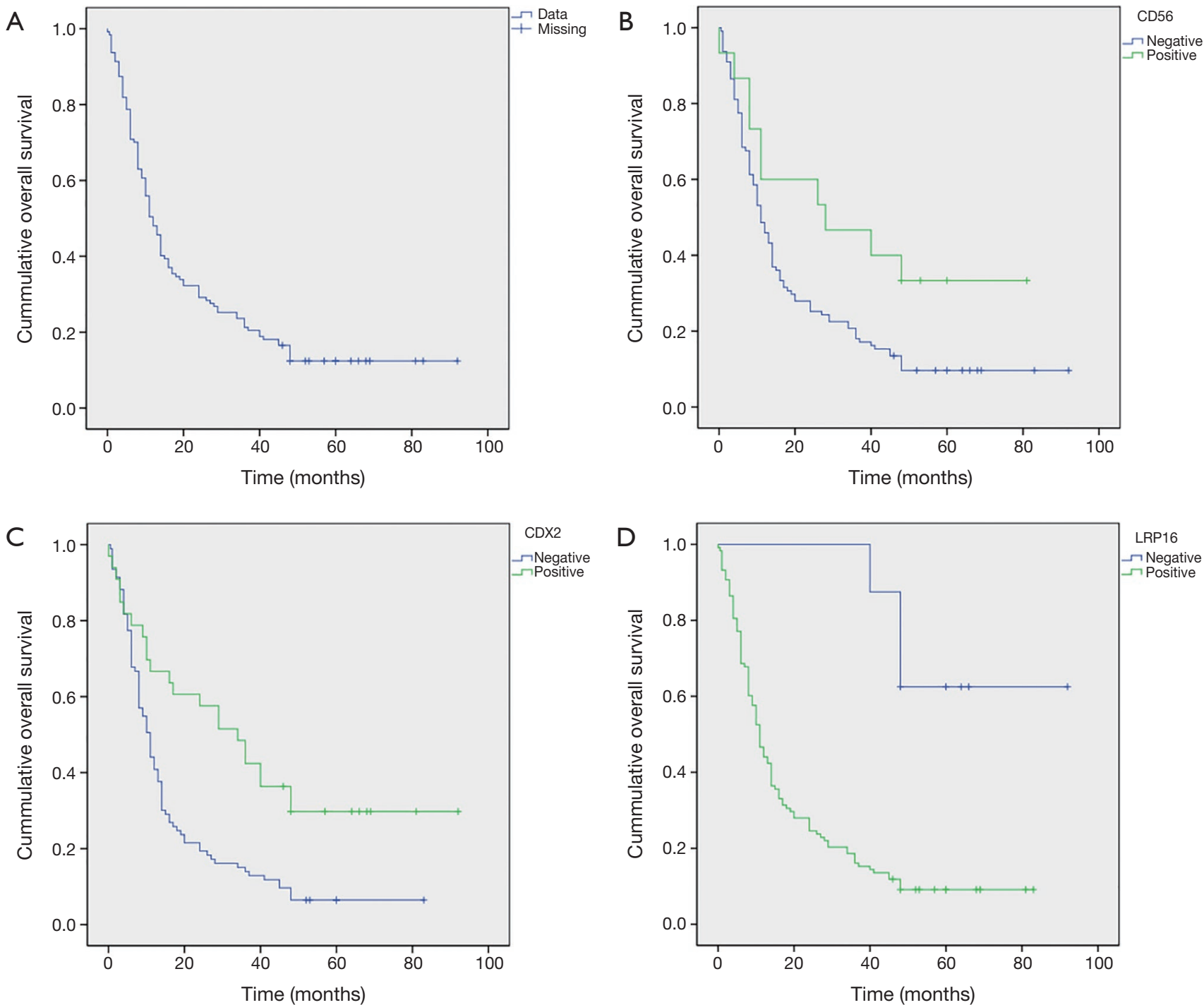

Figure 2 Kaplan-Meier survival analyses. (A) Kaplan-Meier survival analysis of all ICC patients. The y-axis represents the percentage of patients; the $x$-axis, their survival in months. A total of 127 patients were enrolled and followed with a median of 24 months; and 16 patients lost to follow-up. (B) Kaplan-Meier survival analysis by CD56 status. The $y$-axis represents the percentage of patients; the $\mathrm{x}$-axis, their survival in months. The green line represents CD56-positive patients with a trend of longer survival than the blue line representing CD56-negative patients $(\mathrm{P}=0.026)$. A total of 127 patients were enrolled and followed with a median of 24 months; and 16 patients lost to follow-up. (C) Kaplan-Meier survival analysis by CDX2 status. The y-axis represents the percentage of patients; the $\mathrm{x}$-axis, their survival in months. The green line represents CDX2-positive patients with a trend of longer survival than the blue line representing CDX2-negative patients $(\mathrm{P}<0.001)$. A total of 127 patients were enrolled and followed with a median of 24 months; and 16 patients lost to follow-up. (D) Kaplan-Meier survival analysis by LRP16 status. The y-axis represents the percentage of patients; the x-axis, their survival in months. The green line represents LRP16-positive patients with a trend of worse survival than the blue line representing LRP16-negative patients $(\mathrm{P}<0.001)$. A total of 127 patients were enrolled and followed with a median of 24 months; and 16 patients lost to follow-up. ICC, intrahepatic cholangiocarcinoma.

$(\mathrm{P}=0.013)$, gross subtype $(\mathrm{P}<0.001)$, tumor necrosis $(\mathrm{P}=0.001)$, tumor histological differentiation $(\mathrm{P}<0.001)$, TNM staging $(\mathrm{P}=0.012)$, and histological subtype $(\mathrm{P}=0.018)$ were found to be significantly linked with survival. No positive associations were observed between longterm outcomes and age, gender, hepatitis B, diabetes, 
hepatolithiasis, perineural invasion, or vascular tumor thrombus (all $\mathrm{P}>0.05$ ).

\section{Correlation between CD56, CDX2, and LRP16 expression and clinicopathological features}

The pooled result demonstrated a negative correlation between tumor size and CDX2 expression (coefficient of correlation: $-0.190, \mathrm{P}=0.0036$ ), indicating that larger tumors showed a lower expression rate of CDX2. With regard to LRP16, expression was inversely linked with patient age (coefficient of correlation: $-0.183, \mathrm{P}=0.0036$ ). However, LRP16 was positively correlated with tumor differentiation (coefficient of correlation: $0.333, \mathrm{P}=0.001$ ).

\section{Discussion}

The prognostic values of CD56, CDX2, and LRP16 have been documented in certain malignancies previously. However, their predictive significances in ICC yet stay undefined. To the best of our knowledge, the current study serves as the first and largest report to explore the relationships between CD56, CDX2, and LRP16 IHC expressions and survival outcome as well as clinicopathological characteristics in ICC patients. The results illustrated that patients with positive expressions of CD56 or CDX2 had significant survival advantages over those with negative expressions. However, patients with positive expression of LRP16 carried survival inferiority when compared to those with negative expression. Taken together, it conceivable that the above-mentioned molecular markers, particularly LRP16, might be markers of prognostic significances for ICC patients in the future.

The detailed definite mechanisms involved in the correlation between molecular markers' expression and survival influence in ICC patients have not been clearly studied. To some degree, the following aspects could be related to the correlations.

Positive expressions of either CD56 or CDX2 have been proven to be involved in the inhibition of invasiveness and metastasis of ICC, and considered as independent indicators for improved long-term survivals by previous studies $(25,26)$. Previously, Komuta reported that CD56 might be a prognostic marker of cholangiolocellular carcinoma featuring a better prognosis compared with conventional ICC (27). As to cholangiolocellular carcinoma, it has been proven to be a subtype of cholangiocellular carcinoma, and is thought to originate from the ductules/canals of Hering, where hepatic progenitor cells are located (28). Possibly, cholangiolocellular differentiation might be present in certain part of the IHC slides, due to the use of tissue microarray approach. However, it is regarded that cholangiolocellular carcinoma will be defined when more than $90 \%$ of all cells presented cholangiolocellular differentiation (28). Hence, due the predefined exclusion criteria (patients with combined hepatocellular carcinoma and cholangiocarcinoma were excluded from this analysis.), there were no patients with cholangiolocellular carcinoma in our case series. Moreover, in CD56 positive patients, all tumors were adenocarcinoma. Morphological differences were not obviously observed; in these patients, some were with well differentiated adenocarcinoma, and others were with moderately or poorly differentiated adenocarcinoma. But the differences were not significant. As to CDX2, $16.46 \%$ biliary tract carcinoma patients carried positive expression of CDX2 (26). Additionally, it has been demonstrated that CDX2 was expressed in $37.3 \%$ of extrahepatic biliary tract carcinoma and more frequently in tumors with papillary growth and no vascular invasion (29). Commensurate results were also obtained by Jinawath (30). Furthermore, in Li's report, the expression of CDX2 was found to be negatively linked with tumor size and lymph node metastasis; increased CDX2 expression was correlated with better prognostic outcome (31). Similarly, the rate of positive CDX2 expression was $26.2 \%$ in our research; CDX2 expression was also negatively associated with tumor size, but no significant relationship was found between CDX2 expression and morphological tumor subtype. Our study also confirmed the survival benefit of CDX2 positive expression group in comparison with the negative one. On the other hand, LRP16 expression was negatively correlated with CD56 and CDX2 expressions. Herein, patients with positive LRP16 expression were relatively prone to negative expressions of either CD56 or CDX2. And this could partially mirror the prognostic significance of LRP16 expression for ICC. In addition, increased expression of LRP16 gene acts as an ER $\alpha$ or androgen receptor coactivator in tumor related pro-angiogenesis, carcinogenesis and progression, especially for the hormonedependent cancer like breast, endometrial and prostate $(13,16,17,32)$. As to hormone-independent malignancies including gastric and colorectal adenocarcinomas, aberrant expressions of LRP16 were significantly found when compared with corresponding matched noncancerous tissues $(18,20)$. Furthermore, recent research has reflected that LRP16 was regarded as a crucial mediator to the abnormal activation of nuclear factor $\mathrm{kB}(\mathrm{NF}-\mathrm{kB})$ pathway, which has been demonstrated to be extensively involved 
in cell survival and progression of malignancy (33). Taken together, the mechanisms or hypotheses aforementioned might explicate our finding that patients with positive LRP16 expression featured survival inferiority.

Our current work carries certain innovations and strengths. (I) To date, to our best knowledge, LRP16 expression was not widely reported in solid tumors and the present work served as the first trail for addressing the association between LRP16 expression and survival outcomes as well as clinicopathological features in ICC patients. (II) By applying strict patient selection criteria, a total of 127 patients were included and analyzed, forming a large retrospective cohort (especially for a rare disease) from which to make clinical reasonable assumptions about patients.

Despite the mentioned strengths or potential clinical utility, limitation should also be admitted. Namely, the detailed or definite molecular mechanisms implicated in this correlation or process were not explored, thus relatively weakening the conscientiousness and influencing the integrity of our results. Nevertheless, the current study undoubtedly took the first step in developing a persuasive argument for prognostic significance of LRP16 in ICC.

\section{Conclusions}

In conclusion, we evaluated CD56, CDX2, and LRP16 expression by IHC assay in ICC specimens, and found that CD56 positive expression, CDX2 positive expression or LRP16 negative expression implied significant survival benefits for ICC patients. In particular, LRP16 positive expression was commonly identified in ICC and was significantly linked with inferior survival outcomes. Hence, LRP16 expression might provide a new tool to discriminate survival for ICC patients.

\section{Acknowledgments}

The authors thank all colleagues in the Department of Molecular Biology, Chinese People's Liberation Army General Hospital for their help and support with this study. Funding: This work is supported by the grants from the National Natural Science Foundation of China (No. 81472612), Beijing Natural Science Foundation (No. 5162027).

\section{Footnote}

Conflicts of Interest: All authors have completed the ICMJE uniform disclosure form (available at http://dx.doi. org/10.21037/tcr.2018.07.18). The authors have no conflicts of interest to declare.

Etbical Statement: The authors are accountable for all aspects of the work in ensuring that questions related to the accuracy or integrity of any part of the work are appropriately investigated and resolved. The study was conducted in accordance with the Declaration of Helsinki (as revised in 2013). The study was reviewed and approved by the Ethics Committees of the PLA General Hospital (Beijing, China). Informed consent was obtained from the patients.

Open Access Statement: This is an Open Access article distributed in accordance with the Creative Commons Attribution-NonCommercial-NoDerivs 4.0 International License (CC BY-NC-ND 4.0), which permits the noncommercial replication and distribution of the article with the strict proviso that no changes or edits are made and the original work is properly cited (including links to both the formal publication through the relevant DOI and the license). See: https://creativecommons.org/licenses/by-nc-nd/4.0/.

\section{References}

1. Jonas S, Thelen A, Benckert C, et al. Extended liver resection for intrahepatic cholangiocarcinoma: A comparison of the prognostic accuracy of the fifth and sixth editions of the TNM classification. Ann Surg 2009;249:303-9.

2. Casavilla FA, Marsh JW, Iwatsuki S, et al. Hepatic resection and transplantation for peripheral cholangiocarcinoma. J Am Coll Surg 1997;185:429-36.

3. Tang $\mathrm{H}, \mathrm{Lu} \mathrm{W}, \mathrm{Li} \mathrm{B}$, et al. Prognostic significance of neutrophil-to-lymphocyte ratio in biliary tract cancers: a systematic review and meta-analysis. Oncotarget 2017;8:36857-68.

4. Shaib YH, Davila JA, McGlynn K, et al. Rising incidence of intrahepatic cholangiocarcinoma in the United States: a true increase? J Hepatol 2004;40:472-7.

5. Patel T. Worldwide trends in mortality from biliary tract malignancies. BMC Cancer 2002;2:10.

6. McLean L, Patel T. Racial and ethnic variations in the epidemiology of intrahepatic cholangiocarcinoma in the United States. Liver Int 2006;26:1047-53.

7. Bridgewater J, Galle PR, Khan SA, et al. Guidelines for the diagnosis and management of intrahepatic cholangiocarcinoma. J Hepatol 2014;60:1268-89.

8. Tang $\mathrm{H}, \mathrm{Lu} \mathrm{W,} \mathrm{Li} \mathrm{B,} \mathrm{et} \mathrm{al.} \mathrm{Influence} \mathrm{of} \mathrm{surgical} \mathrm{margins}$ on overall survival after resection of intrahepatic cholangiocarcinoma: A meta-analysis. Medicine 
(Baltimore) 2016;95:e4621.

9. de Jong MC, Nathan H, Sotiropoulos GC, et al. Intrahepatic cholangiocarcinoma: an international multiinstitutional analysis of prognostic factors and lymph node assessment. J Clin Oncol 2011;29:3140-5.

10. Kim JH, Won HJ, Shin YM, et al. Radiofrequency ablation for the treatment of primary intrahepatic cholangiocarcinoma. AJR Am J Roentgenol 2011;196:W205-9.

11. Xu HX, Wang Y, Lu MD, et al. Percutaneous ultrasound-guided thermal ablation for intrahepatic cholangiocarcinoma. Br J Radiol 2012;85:1078-84.

12. Kiefer MV, Albert M, McNally M, et al. Chemoembolization of intrahepatic cholangiocarcinoma with cisplatinum, doxorubicin, mitomycin C, ethiodol, and polyvinyl alcohol: a 2-center study. Cancer 2011;117:1498-505.

13. Han WD, Mu YM, Lu XC, et al. Up-regulation of LRP16 mRNA by 17beta-estradiol through activation of estrogen receptor alpha (ERalpha), but not ERbeta, and promotion of human breast cancer MCF-7 cell proliferation: a preliminary report. Endocr Relat Cancer 2003;10:217-24.

14. Han WD, Yu L, Lou FD, et al. Cloning and expression characterization of the full length cDNA for a novel leukemia-associated gene LRP16. Zhongguo Shengwu Huaxue yu Fenzi Shengwu Xuebao 2001;17:209-14.

15. Yu L, Han WD, Lou FD, et al. Cloning of leukemia associated gene LRP16 in acute myeloid leukemia. Junyi Jinxiu Xueyuan Xuebao 2000;21:81-4.

16. Han WD, Mu YM, Lu XC, et al. Estrogen stimulates human breast cancer MCF-7 cell proliferation by up-regulation of LRP16 mRNA via activation of estrogen receptor-alpha. Zhonghua Neifenmi Daixie Zazhi 2004;20:165-8.

17. Han WD, Zhao YL, Meng YG, et al. Estrogenically regulated LRP16 interacts with estrogen receptor alpha and enhances the receptor's transcriptional activity. Endocr Relat Cancer 2007;14:741-53.

18. Xi HQ, Zhao P, Han WD. Clinicopathological significance and prognostic value of LRP16 expression in colorectal carcinoma. World J Gastroenterol 2010;16:1644-8.

19. Shao $Y, \mathrm{Li} X, \mathrm{Lu} Y$, et al. Aberrant LRP16 protein expression in primary neuroendocrine lung tumors. Int $\mathrm{J}$ Clin Exp Pathol 2015;8:6560-5.

20. Li YZ, Zhao P, Han WD. Clinicopathological significance of LRP16 protein in 336 gastric carcinoma patients. World J Gastroenterol 2009;15:4833-7.

21. Zhao P, Lu Y, Han W. Clinicopathological significance and prognostic value of leukemia-related protein 16 expression in invasive ductal breast carcinoma. Cancer Sci 2010;101:2262-8.

22. Hidalgo A, Pina P, Guerrero G, et al. A simple method for the construction of small format tissue arrays. J Clin Pathol 2003;56:144-6.

23. Tang H, Lu W, Yang Z, et al. Significance of incorporation of DNMT1 and HLA-DR $\alpha$ with TNM staging in patients with hepatocellular carcinoma after curative resection. Int J Clin Exp Pathol 2017:9372-81.

24. Kammerer U, Kapp M, Gassel AM, et al. A new rapid immunohistochemical staining technique using the EnVision antibody complex. J Histochem Cytochem 2001;49:623-30.

25. Chiu CT, Chiang JM, Yeh TS, et al. Clinicopathological analysis of colorectal cancer liver metastasis and intrahepatic cholangiocarcinoma: are they just apples and oranges? Dig Liver Dis 2008;40:749-54.

26. Chang YT, Hsu C, Jeng YM, et al. Expression of the caudal-type homeodomain transcription factor CDX2 is related to clinical outcome in biliary tract carcinoma. J Gastroenterol Hepatol 2007;22:389-94.

27. Komuta M, Govaere O, Vandecaveye V, et al. Histological diversity in cholangiocellular carcinoma reflects the different cholangiocyte phenotypes. Hepatology 2012;55:1876-88.

28. Komuta M, Spee B, Vander Borght S, et al. Clinicopathological study on cholangiolocellular carcinoma suggesting hepatic progenitor cell origin. Hepatology 2008;47:1544-56.

29. Hong SM, Cho H, Moskaluk CA, et al. CDX2 and MUC2 protein expression in extrahepatic bile duct carcinoma. Am J Clin Pathol 2005;124:361-70.

30. Jinawath A, Akiyama Y, Yuasa Y, et al. Expression of phosphorylated ERK1/2 and homeodomain protein CDX2 in cholangiocarcinoma. J Cancer Res Clin Oncol 2006;132:805-10.

31. Li QL, Yang ZL, Liu JQ, et al. Expression of CDX2 and hepatocyte antigen in benign and malignant lesions of gallbladder and its correlation with histopathologic type and clinical outcome. Pathol Oncol Res 2011;17:561-8.

32. Zhao YL, Han WD, Li Q, et al. Mechanism of transcriptional regulation of LRP16 gene expression by 17-beta estradiol in MCF-7 human breast cancer cells. J Mol Endocrinol 2005;34:77-89.

33. Wu Z, Li Y, Li X, et al. LRP16 integrates into NF-kappaB transcriptional complex and is required for its functional activation. PLoS One 2011;6:e18157.

Cite this article as: Wang $Z$, Tang $H$, Yuan J, Song $X$, Song $Z$, Zhao P. The expression and clinicopathological significance of CD56, CDX2, and LRP16 in intrahepatic cholangiocarcinoma. Transl Cancer Res 2018;7(4):1036-1043. doi: 10.21037/tcr.2018.07.18 